\title{
CIRCULAR NO. 11.
}

(MARCH, 1904.)

\section{FUMIGATION PRACTICE.}

BY C. W. WOODWORTH.

There have been so many demands for information upon the practice of Orchard Fumigation that the edition of Bulletin No. 122 on that subject has been entirely exhausted. The present circular, condensed from that bulletin, is intended to furnish this information as to the details of the process, with suggestions of possible improvements.

All effective fumigation is done in inclosed spaces, usually in cloth tents. This is necessary on account of the nature of gas, that of diffusing itself through the air, which makes it so efficient in reaching every insect on the tree. Unless inclosed, it would quickly become diluted and fail to do effective killing.

The Canvas.-Common duck is now uniformly employed for making the larger tents, most of them being made of the eight-ounce canvas, such as is used for light sails. Six-ounce drilling is often used for the smaller tents. The cloth is lapped and double-sewed in the same manner as for tents or sails. The edge is usually simply hemmed, but some bind it with rope. Whenever permanent rings for handling are attached, the tent is reinforced, but this is a matter in which there is much diversity. The details of the construction will depend somewhat on the size and kind of tent, and will be referred to again, below.

After the making of the tent, it was formerly the practice to treat it in some manner to make it gas-tight, so as to confine the gas better. Some fumigators still treat their tents. Four methods are used for this purpose, all of which seem to give good satisfaction.

The first method is to thoroughly treat the tent with boiled linseed oil. It is applied freely with a brush, and the whole cloth becomes saturated with it. The tent must be kept spread out until quite dry, for the oil has a great tendency to heat if not exposed freely to the air, and the cloth chars and becomes rotten. If properly done, the tent remains strong and tight, and is not too stiff.

The second method consists in the use of sizing and paint. The 
sizing is applied in the same manner as the oil, and penetrates the fiber of the cloth in the same way. As soon as this coat is dry it is followed by another of rather thin flexible paint, sometimes on both sides; the result being a perfectly tight tent with a very smooth surface and fully as flexible as the oiled tent. The sizing protects the fiber of the cloth, so there is no danger of heating.

The third method is the saturation of the cloth by a decoction of the chopped-up leaves of the common prickly pear cactus (Opuntia engelmani). This decoction is made by filling a barrel two-thirds full of the chopped stems, adding cold water until the barrel is nearly full; then letting it soak for twenty-four hours, when it is drawn off and strained, and is ready for use. This decoction is seldom used by itself, but other substances are added according to the whim of the person treating the tents. Very generally a pigment, like yellow ochre or Venetian red, is added to give more body to the mixture; sometimes glue is added also. In tents treated with the cactus decoction, there is some tendency to become moldy when not in use, to prevent which some prepare a tannin solution to add to the mixture. The decoction may be applied to the tents with a brush, but a better way is to soak them during the night in a trough containing the mixture. In the morning they can be raised by means of ropes and pulleys and allowed to drain for some time and then spread out to dry. Tents treated with this mixture are scarcely at all stiffened and seem to be satisfactorily tight.

The fourth method is to soak the canvas in a solution of tannin until it assumes a rich brown color. This method does not make the tent as tight as those described above, but is quite as often used as any.

The Bell Tent.-The tents known as bell tents are cylindrical in shape, with the top rounded over like a dome. They are used in connection with a derrick, by means of which they are placed upon and lifted from trees; the derrick also supports the weight of the tent while it is upon the tree. The bell tent was one of the original forms of tents, and while mostly supplanted by other styles, is still used to a considerable extent, especially for very large trees. It is the only form of tent now in use where the whole weight of the tent is not carried by the tree, and many favor it for this reason.

The derrick used with the bell tents at the present time is that used with the Preble fumigator, or some modification of it. This is shown in Fig. 1. It consists of a wagon, which supports a mast considerably higher than the trees to be fumigated, and is braced at the bottom with stays that hold it rigidly in place. Across the top of the mast a yard is fastened and braced with trusses extending from the mast. The length of the yard is about a third longer than the distance between the rows of trees. Near each end of the yard are placed cross-bars as shown 
in the illustration. The arrangement of the ropes can be understood from a study of the figure.

The heaviest rope is attached to the top of the tent with double pulleys. Along the lower edge, on the four sicles of the tent, are fastened boards, generally of ordinary six-inch fencing, which are called trail boards, and from the center of each of these the trail ropes pass upward and over pulleys attached to the yard and ends of the cross-bars. All these ropes follow the yard until near the mast, then passing again over pulleys, they go down to the bed of the wagon and are fastened over belaying pins. The trail ropes pass through thimbles along the side of the tent as well as through the pulley at the center of the trail, so that when the latter is drawn up to the yard or cross-bars, the sides of the

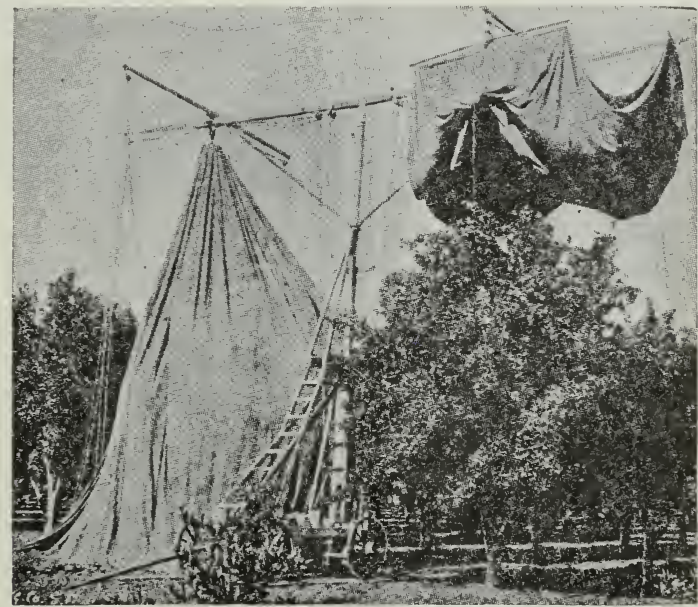

FIG. 1. The Bell Tent-Derrick with one tent nearly in place on a tree, and the other drawn up ready for moving.

tent are gathered in three or four places and raised almost as high. The only other ropes are the guide lines attached to the center of the trails and hanging free. They are of such length as to reach the ground when the tent is elevated.

The manipulation of these tents can be readily understood from a study of the engraving. While the tree is being fumigated the tent is usually allowed to rest partly on the tree, and not drawn up to the yard as shown in the illustration. Two persons can handle the apparatus, but three or four greatly facilitate the work. The procedure in changing the tent is as follows: Supposing that both tents are upon the trees and the time has arrived to make the change, the first operation is to pull on the main rope attached to the center of the tent and raise this as far as it will go easily, and then fasten the rope again to the belaying 
pin. If short-handed, one tent is raised at a time, but with plenty of help both go up at the same time. The trail ropes are next taken in hand and pulled all together, and if this becomes difficult, two (or even one at a time) are pulled until the tent on all sides is pulled up to the yard and cross-bars. While this is going on one person (or perhaps more) is kept busy seeing that the tent is clearing the tree properly. His first business is to see that the edge with the trail boards is not caught inside of the tent; it should slip up around outside of it. Later he will be occupied with making the tent slip off the projecting branches. He can generally do this by pulling on the guide lines, but on very large trees he may find a light ladder necessary. The removal of the tent would be comparatively easy but for the work at the ropes. After all the ropes are pulled tight, including the main rope, and both tents are against the yard, the apparatus is ready to shift to the next row. The wagon may be pulled along by hand, or by a horse hitched to the end of the tongue. If the ground is a little uneven, the apparatus can be kept from tipping over by steadying it with the guide lines. Arriving at the proper position between the next two trees, the first thing is to arrange the guide lines in their places around the tree. The trail ropes are now released and the tent is allowed to slowly descend upon the tree. While this is taking place, one or more are busy with the guide lines, pulling the trail boards this or that way as may be necessary to clear the branches. If a branch is particularly spreading it may be necessary to use a ladder, forcing it within the tent by hand. Should the trees be very large the branches will extend over the wagon, causing much trouble in pulling the tent down on that side. With a small, symmetrically shaped tree the tent can be lowered rapidly into place without any trouble whatever. After the trail ropes are all played out, the main rope is loosened and the tent allowed to settle to the position desired, and fastened there. There yet remains the job of seeing that the tent is tight to the ground on all sides. The trail boards are made to lie on the part of the tent that is on the ground, and earth is thrown on any part of the edge of the tent that does not lie down well. When both tents are thus in position they are ready for the "fumigator," or" man who charges the generator.

The Hoop Tent.-The form most used in this State is the hoop tent, which is a development from the bell tent and is of the same general shape. The hoop was first used as a means of keeping the mouth of the bell tent open, but it was soon discarded in favor of the trail boards. It was, however, discovered that for rather small-sized tents the hoop afforded a better means of handling than did the derrick.

The hoop tents now in use range from 8 to 14 feet in diameter. They are made in the same way as a bell tent, omitting, however, the arrange- 
ments for suspending them, and possessing, instead, a series of cloth loops for attaching the hoop, as is shown in the engraving.

The hoop is usually made of $\frac{3}{4}$-inch gas pipe; $\frac{1}{2}$-inch pipe will do for

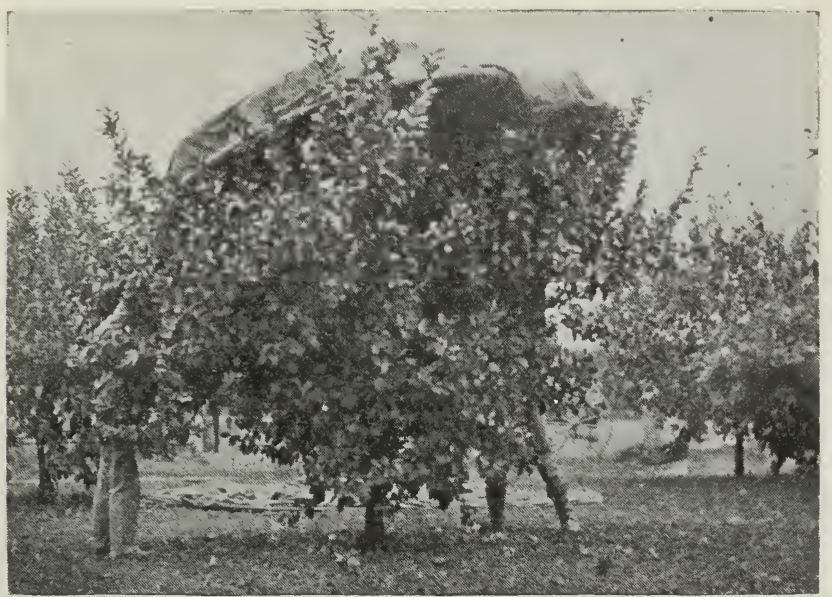

FIG. 2. The Syall Hoop Text-Throwing the tent over a tree.

the smaller sizes, but it is too weak for hoops above 10 feet in diameter, as it bends too easily and soon becomes very crooked. To make the hoop, pipe is coupled together until the proper length is reached accord-

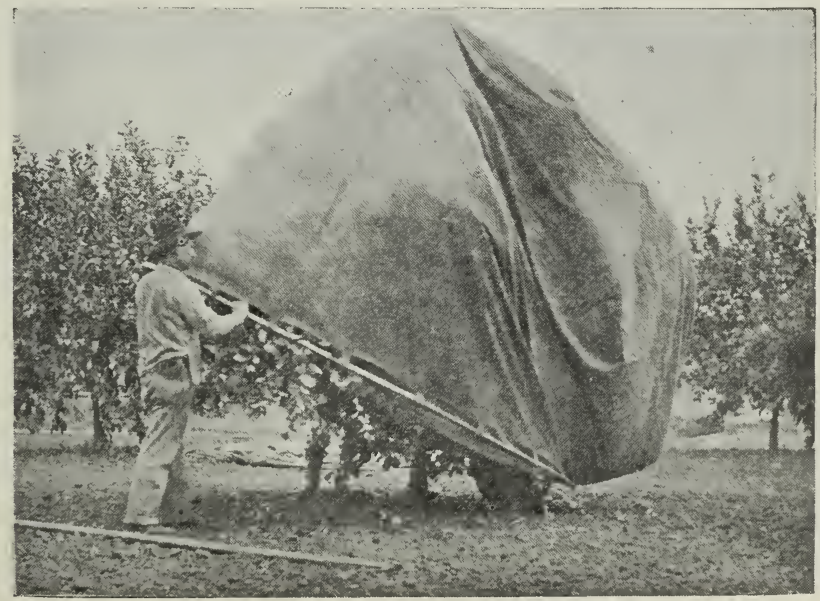

FIG. 3. The SHall HoOp Text-pulling down the tent.

ing to the size desired, and then bent into shape. The union is then made by inserting into the ends a piece of iron rod a foot or less in length and just small enough to enter the pipe. Holes are now drilled 
through the pipe and rod, and rivets are inserted, thus making the joint fast. A coupling with right- and left-hand threads might be used instead of the rod and rivets.

The manipulation of a hoop tent varies according to its size. When the diameter of a tent is not much greater than the distance between

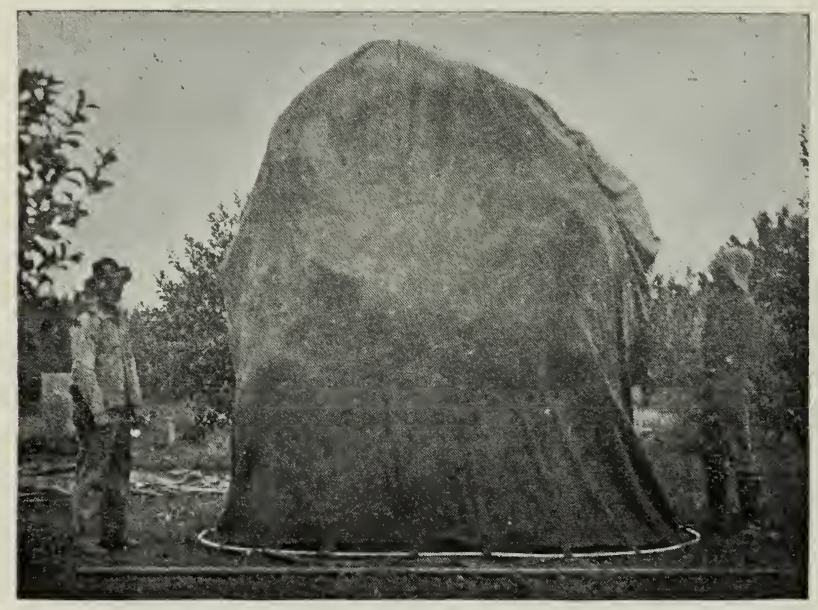

FIG. 4. The Siall Hoop Tent-Ready for the fumigator.

the nearest branches of adjacent trees, the procedure is that illustrated in the diagram, Fig. 5, and depicted in Figs. 2-4.

To move such a tent from one tree to the next, two men place themselves on opposite sides of it, grasp the hoop and raise the side which is

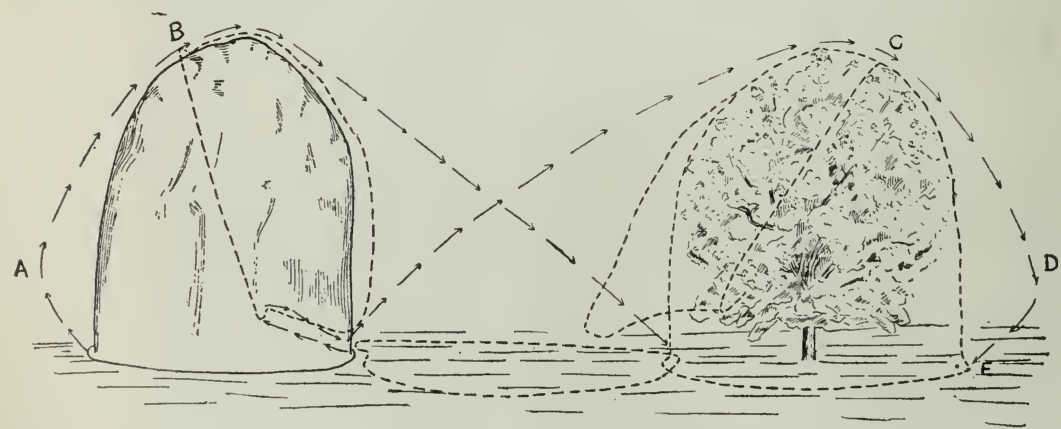

FIG. 5. Diagram illustrating the method of shifting a small hoop tent from one tree to another. The letters indicate the successive positions of the hoop as the tent is first thrown off of one tree on to the ground, and then is picked up and put over the next.

opposite the tree to which they intend to move it; they step sidewise, dragging the side that is on the ground closer to the trunk, and the hoop will be in about the position indicated by $\mathrm{A}$ in the diagram. The men, still holding the hoop as they first grasped it, continue to raise the free side until it passes over the top of the tree, when it is allowed to 
fall to the ground between the two trees. In falling, the hoop naturally moves away from the tree from which it came, so that the cloth falls orer the edge of the hoop, as shown in the diagram. If this does not

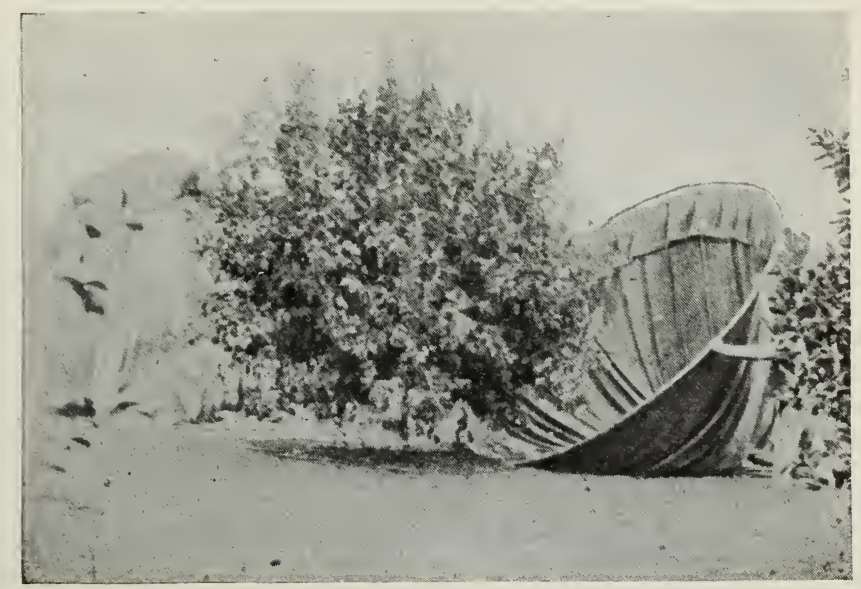

FIG. 6. The Large Hoop Tent-Putting the tent on a tree. The tent in this case is being lifted from the ground, and not shifted from another tree.

occur, the tent is pulled into that position in order that, when the hoop is raised, the center of the tent will be brought at once to about the center of the top of the tree.

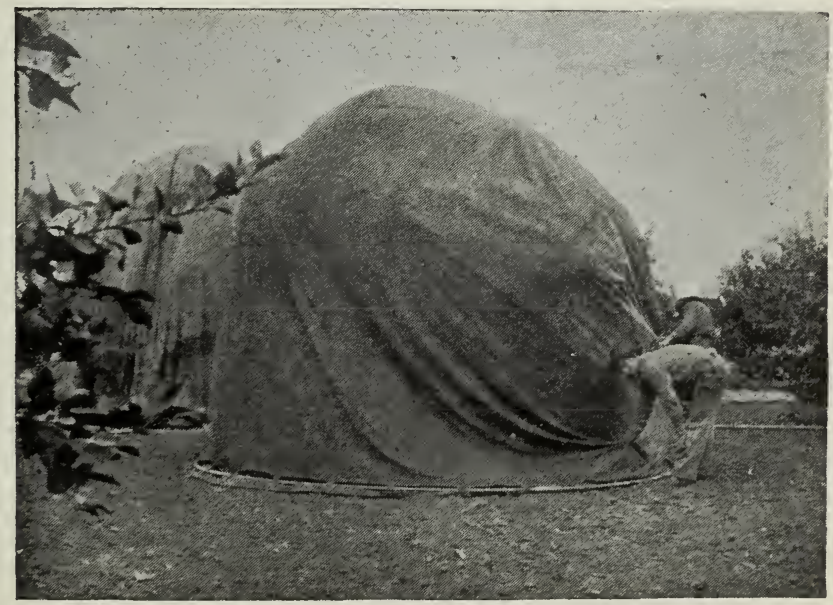

FIG. 7. The Large Hoop Tent-Pulling down the canvas so that the hoop will rest freely on the ground.

The men now grasp the hoop again, as before, carry it.toward the tree and lift up the farther edge, then with one movement throw it over the tree to about the position indicated by $\mathrm{D}$. Often it will go clear to the 
ground and needs no further attention. If it stays at D, the men proceed to the point highest from the ground, and pull on the hoop and canvas until the former rests easily on the ground. The cloth which

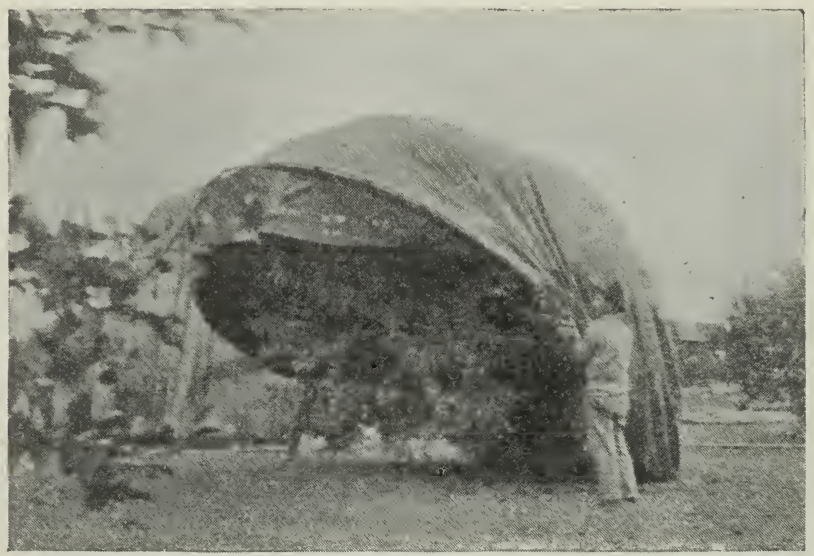

FIG. 8. The Large Hoop Text-Beginning to raise the tent

extends beyond the hoops forms a sufficiently tight contact with the ground if the latter is ordinarily level.

The manipulation of the large hoop-tents differs from that above described, from the fact that the proximity of the trees makes it im-

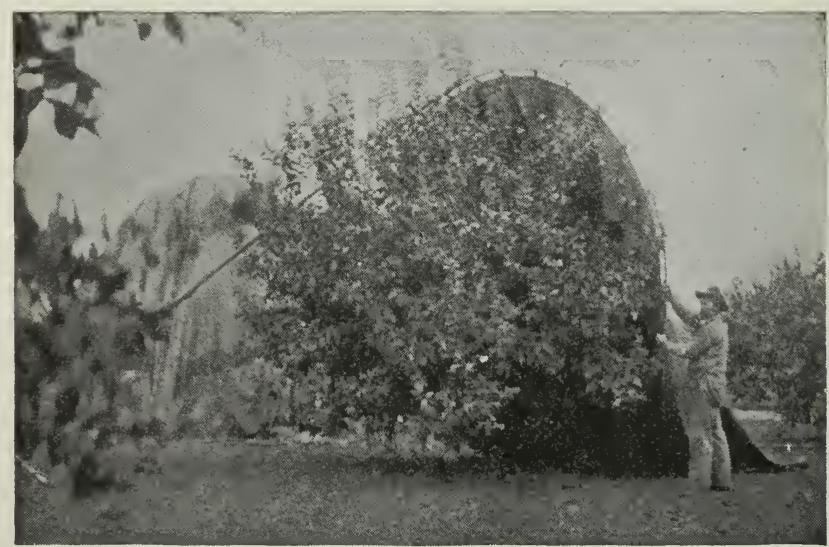

FI(i. 9. The LiRge Hoop Tent-The tent almost off the tree.

practicable to lay the tent on the ground. The procedure in this case is indicated in the accompanying diagram, Fig. 10, and by Figs. 6-9.

It is better to have three men to handle these tents, although two can do it. When working three, two take hold in the same way as described 
above for the small hoop tents, and the third pulls on the side that is raised to the position $\mathrm{A}$. The latter then catches the hoop with a fork at the end of a pole, and as the others lift he assists by pushing. This is shown in Figs. 8 and 9.

When the hoop has taken about the position shown at B, in Fig. 10, or a little past that point, the two men holding the sides of the tent carry it to the next tree to the position $\mathrm{C}$, and then without pausing, and while the tent is full of air and streaming out behind with the aid of momentum acquired, the upper edge of the hoop is forced over the top of the tree and down on the other side. Generally it is possible to throw the hoop into the position $\mathrm{D}$, when it can readily be pulled dow to the ground

If there is any trouble in pulling the cloth over, the third man,

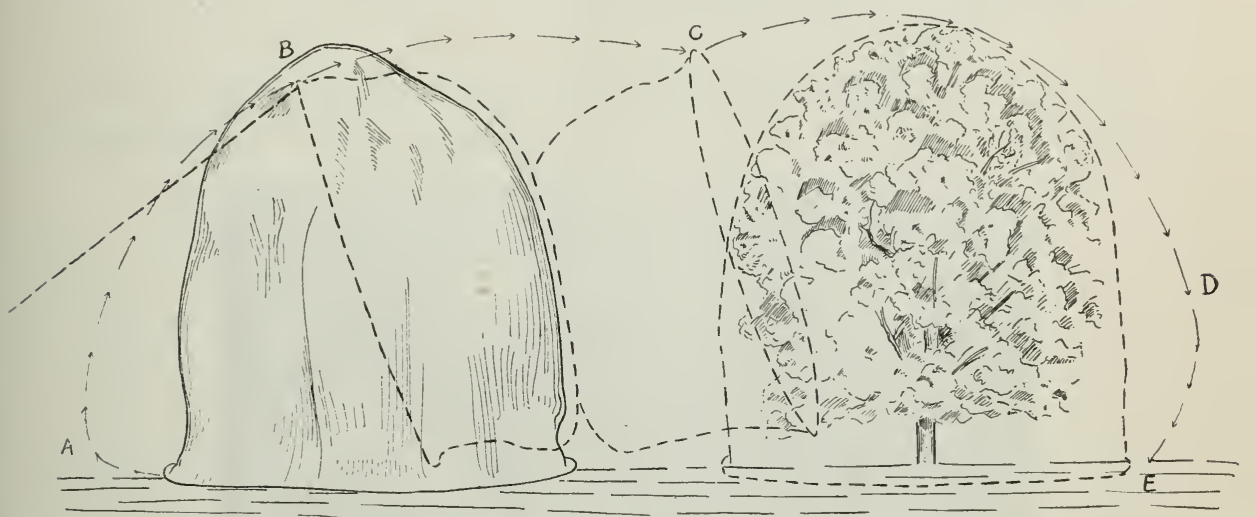

FIG. 10. Diagram illustrating the method of shifting a large $h_{1}$ oop tent from one tree to another. The letters indicate the successive positions of the hoop.

having tossed his pole to the next tent, goes around to the near side of the tent just moved, and as the others pull on the far side, shakes the cloth of the tent away from the tree, thus relieving some of the friction. The weight of the hoop of these large tents greatly helps in the process of slipping the cloth over the tree, the most energy being required in removing the tent. The large tents are moved quite as rapidly as are the smaller ones. It will be noticed that the cloth is turned inside out with each change in the case of the larger tents, but with the smaller ones the same side of the cloth is always next to the tree.

Method of Cutting Cloth for Bell Tents.-All of these tents are made in the same manner, and are the most economical in cloth of any tents made. Commonly the tent is made by the "cut and fit" method. These tents may be made with scarcely any loss, if cut according to the following directions: Measure off strips of a length equal to twice the height plus one tenth the diameter of the tent desired. These will make 
two strips each by marking the exact middle and measuring off on one edge from the middle line one quarter of the diameter of the tent and on the other one half the diameter. Now take a long strip of molding and bend it so as to touch these three poinits and mark off the curve so

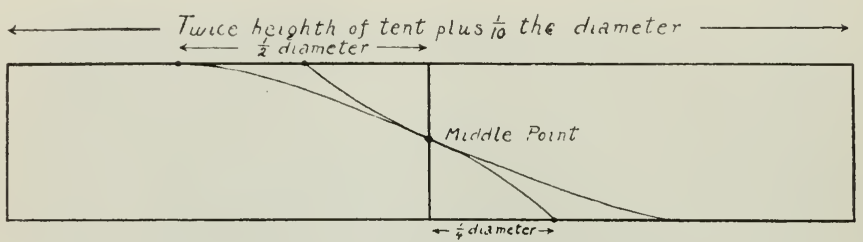

FIG. 11. Method of cutting cloth for bell tents.

produced. This allows for the seam. In making up, sew the two cut edges together in each pair of strips. The accompanying figure will assist in making these directions plain.

The Box Tent.-A recent development along this line is what is known as the box tent. It is an Eastern idea devised for use on deciduous

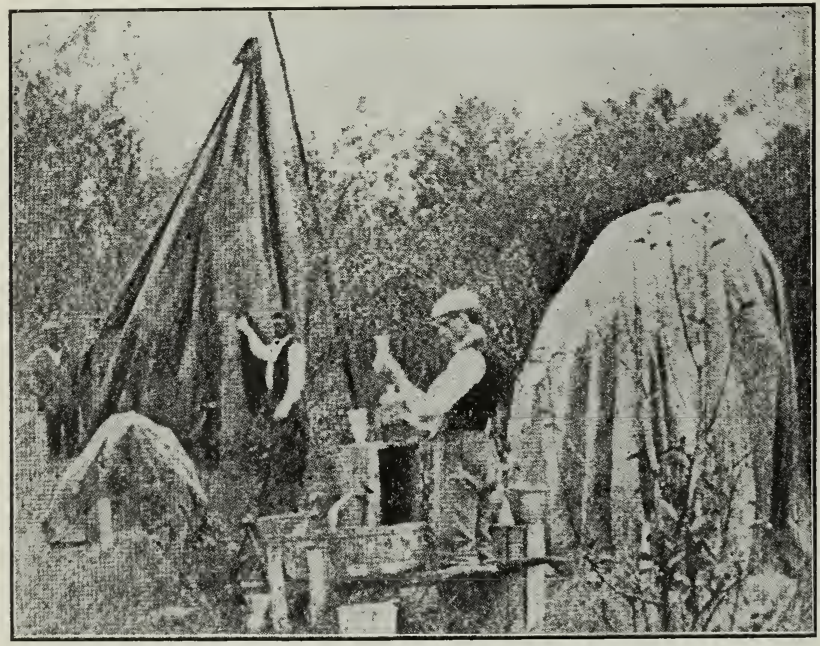

FIG. 12. The Box Tent-Lifter in position over a tent ready to remove it from the tree.

trees, in which the wood is less pliable and more easily broken than is that of citrus trees. It may deserve a trial in our orchards, although, doubtless, we would soon simplify the handling so as to work more rapidly than is now possible.

The box tent is somewhat intermediate between the bell, or hoop tent, and a sheet tent. It has something of the shape of the hoop tent, but flaring beneath and without anything stiff at the bottom. It is made with a square top-piece and with four sides, which are a half larger at the bottom than at the top. 
The manipulation of this tent is accomplished by the use of a pole called the "lifter." This pole has a piece of scantling fastened to the bottom and braced, as shown in Fig. 12, and in the accompanying diagram, Fig. 13. To the top of the pole is attached a guy rope and a block pulley, over which passes a rope for lifting the tent. A small Y at a convenient height for fastening the rope completes the lifter.

In changing the tent from one tree to another the process is to place the lifter next to the tree to be uncovered. The guy rope is then tied to an adjacent tree in such a manner as to allow the end of the lifter to stand over the center of the tent. The end of the lifter rope is fastened to the edge of the tent opposite the base of the lifter. As the

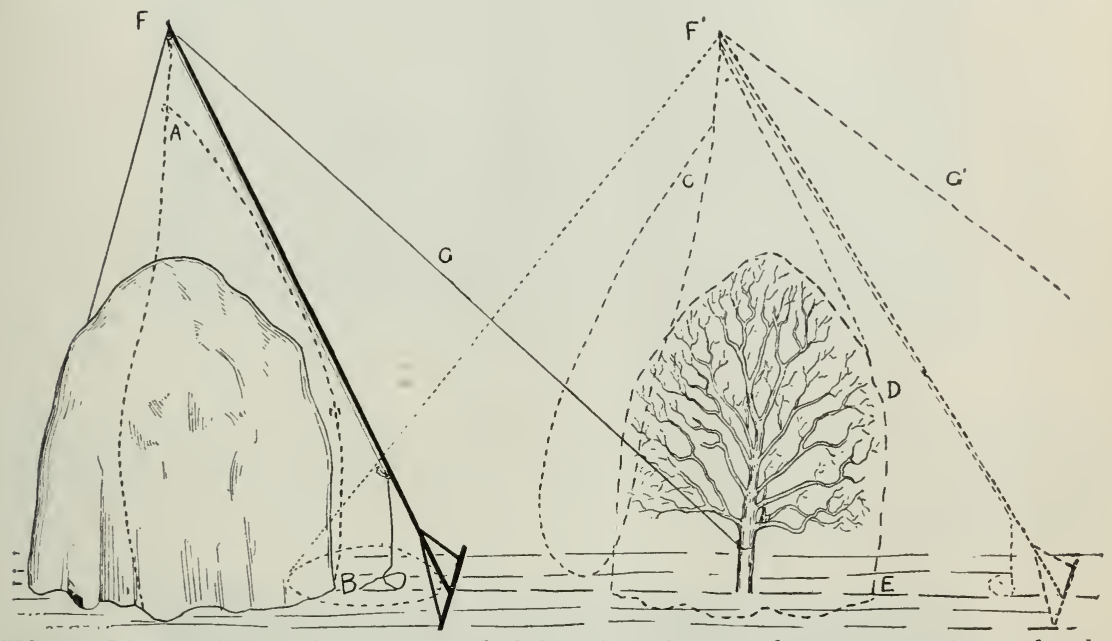

FIG. 13. Diagram illustrating the method of changing a box tent from one tree to another by the use of the lifter. A, B, C, D, and E represent the successive positions of the tent; F, the lifter; $\mathrm{G}$, the guy rope; $\mathrm{F}^{\prime} \mathrm{G}^{\prime}$, the second position of lifter and guy rope.

edge of the tent is being lifted by this rope, assistants pull the edge of the tent aside, freeing the branches; small forked poles are used to assist in this work. After all the branches are free the tent is lowered to the ground beside the tree.

The lifter is now moved to the tree over which the tent is to be placed, as shown at C. By pulling on the lifter rope, the edge of the tent is raised again to the top of the pole, when the rope is fastened. Two persons now take hold of the edges of the tent and pull them around the tree, while a third pulls on the guy rope until the top of the tent is over the top of the tree, when the tent is lowered and pulled into position. The movement of the lifter by means of the guy rope is not indicated in the drawing, but can be readily understood without. The same movement is useful in untenting a tree.

After letting the tent down over the tree the lifter rope is untied and the bottom of the tent pushed up toward the tree and made to lie close 
to the ground. If necessary, a little earth is thrown upon it to hold it down.

The Sheet Tent.-The form that seems. most likely to replace all others, and has now indeed almost done so, is the sheet tent. It is the simplest to make, the most readily adaptable to all sizes of trees, and

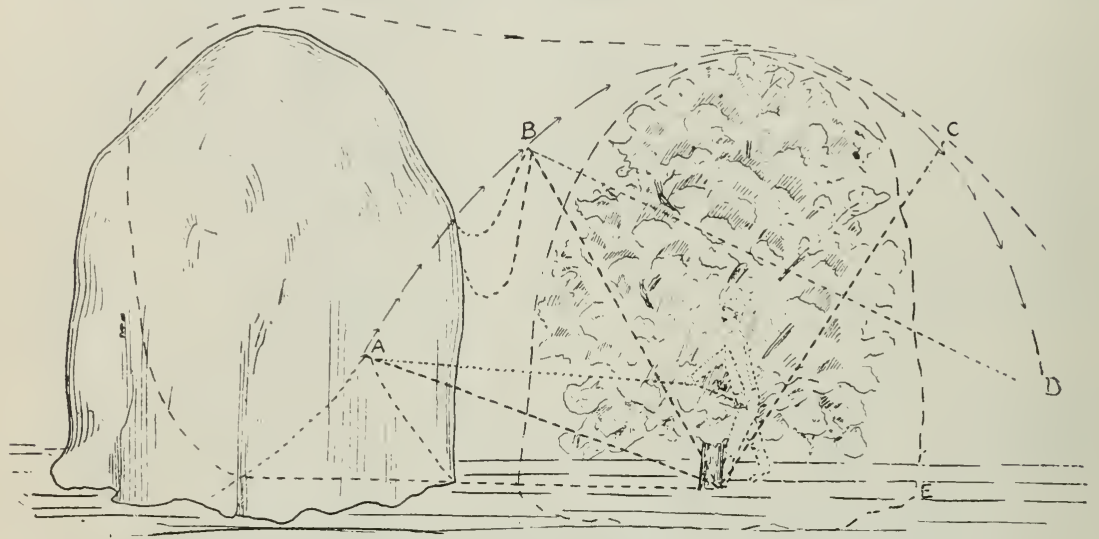

FIG. 14. Diagram illustrating the method of shifting a sheet tent from one tree to another. The letters represent the successive positions of the end of the pole.

is almost as readily moved from tree to tree as the hoop tent. It contains, however, a great deal of useless canvas, which is an objection to the economical mind.

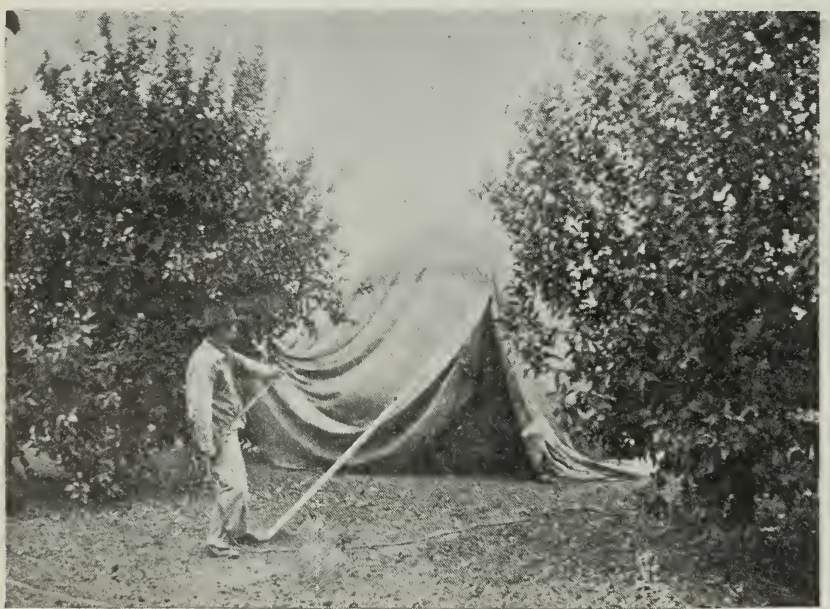

FIG. 15. The Sheet TExt-Beginning to lift the edge of the tent.

Sheet tents are made either in a regular or in an oval hexagon, and perfectly flat. A pair of rings is often attached on each side, near what is intended as the front edge; it is convenient to attach these rings by 
iron links, so that they can be rattled and found in the dark by shaking the tent.

The movement of the tent is accomplished by the use of two poles.

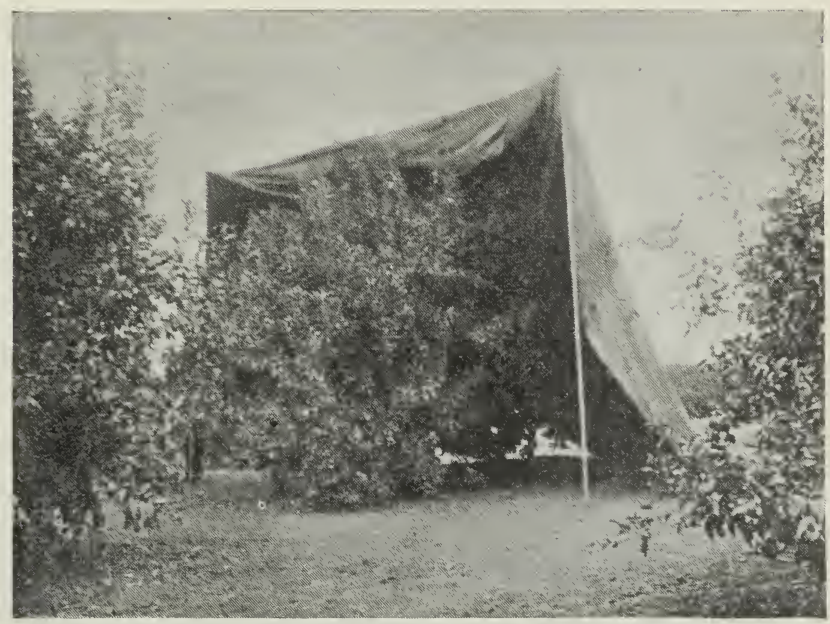

FIG. 16. The Shen T Tent-The tent partly over the next tree.

These are usually simple poles with a small rod projecting from the upper end, over which the ring of the tent is slipped; a rope is also fastened at the upper end. The length of the pole is slightly greater

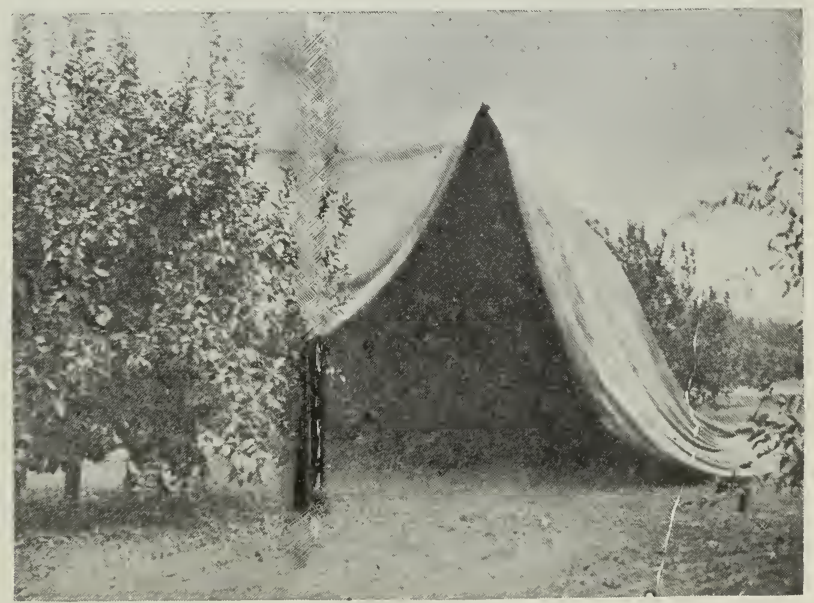

Fifr. 17. The Sheet Tent-Tree almost covered, pole falling.

than the height of the trees it is desired to cover. Sometimes the pole has the same shape as the lifter used for the box tents, but the pulleys and guy ropes are not needed, except for the largest trees. 
The ordinary process of moving the sheet tent is shown in Figs. 15-18 and by the accompanying diagram, Fig. 14. A bird's-eye view is also shown in Fig. 19. The men approach the tent to be moved, poles in hand, and finding the rings insert the small rods at the end of the poles and take a hitch with the rope over the ring to prevent the latter from slipping off. They then proceed to the other end of their poles, which they have placed even with the trunk on opposite sides of the tree to which the tent is to go. While taking this station they have not let go of the rope, but have held it tight enough not to loosen the tent ring. The next step in the process is to place one foot on the end of the pole to prevent it from slipping, and to pull on the rope. This will lift up the edge of the tent as shown at $\mathrm{A}$ in Fig. 14. As the men continue to pull on the rope the end attached to the tent moves through the arc

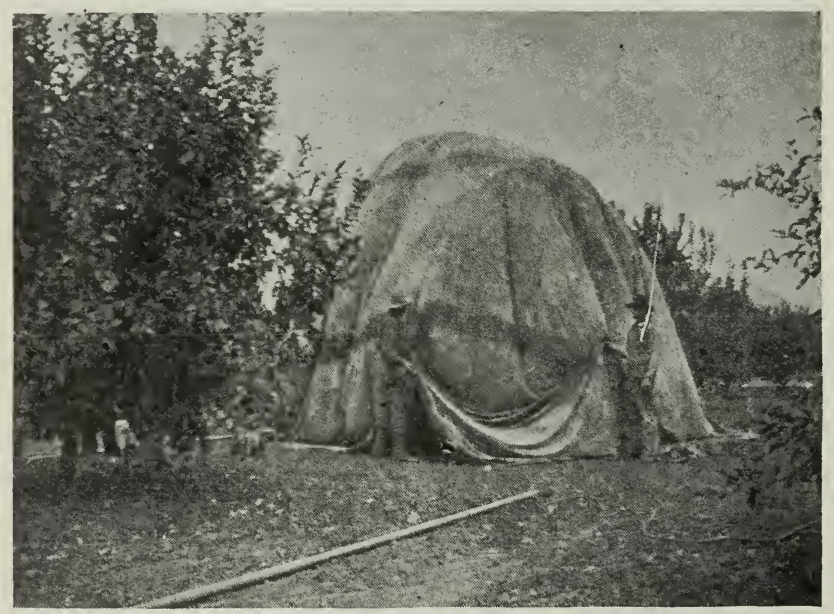

FIG. 18. The Sheet Tent-Adjusting the bottom of the tent.

indicated by the line of arrows. As soon as the pole becomes nearly enough upright so as not to slip when the foot is removed from the end, the man backs off, away from the tree, and thus gets a more direct pull on the tent, which by this time has begun to require some considerable effort. This becomes necessary also in order that the pull from each side may stretch out the front edge of the tent so that it may clear the top of the tree.

The tent is now spread out over two trees and reaches the ground on either side. As the men at the ropes continue to back away the tent is slipped from one tree to the next and the poles fall to the ground. In this last stage in the process care must be taken that both poles reach the ground at about the same time. If this is not done the tent will shift to the side of the pole which first reaches the ground, and if that side is 
pulled very much too fast the tent may not reach the ground on the opposite side, and sheet tents are rather harder to adjust than other kinds. This same difficulty, in regard to the front and back ends of the tent, often occurs when using a tent barely large enough for the tree. If the tent is pulled too slowly the poles will slip when the tent is not quite over, and the front will not reach the ground; and on the other hand, if it is pulled too rapidly, the tent will go too far, and the back end be free from the ground. The oval tent was made to overcome this difficulty, for with it care only need be taken to slide the tent far enough.

When using a large tent for a very small tree the tent is pulled up so as to have sufficient slack canvas to go over the tree, and this is pulled over by hand. When being removed, the cloth is pulled back in the same manner as it was put on, and dragged along the ground to the next tree.

In the case of very large trees, which require the lifter style of pole, the process is as follows: The poles are set up and the guy ropes attached as described for the box tent, only that two poles are used. The other ropes are now attached to the tent at the near edge and the latter pulled to the top of the pole. The rope is then made fast, the guy ropes pulled, and the tent slid in the same manner as with smaller tents. Sometimes the pole is not set at such an angle, but nearer the tent, when it will be necessary, after sliding the tent part of the way, to again tie the guy rope and lift the bottom of the pole over; it will then be opposite the trunk, and the tent will be lifted high enough when it is given the final shift.

When there is fear of breaking the branches in removing a tent, the practice is to "skin it off," using a pole of the lifter pattern, and carry the rope around to the far side and attach it to the edge of the tent there. The tent by this method slides over itself and saves the tree to that extent; it is pulled over on to the next tree as in the preceding methods. Since much of the tent by this method falls to the ground, it is harder on the tree while it is being tented. By this process the tent is reversed each time it is changed.

Paper Tents.-Eastern practice has developed a number of forms of tents of fixed size with wooden frames covered with paper. 'These are probably the tightest tents in use, but are not adjustable to varying sizes of trees. They are usually rectangular in shape. The frame is made of light wooden strips well braced, and the paper used is ordinary building paper of rather good quality. This is tacked on to the frames. They can only be used for trees under eight feet or thereabouts in height, except with the use of derricks, which makes the operation rather slow.

A form of paper tent open on the side, with a system of clamps for 
making all tight after the door is closed, is satisfactory in every way except in speed.

One great adrantage of tents of this sort is the uniform volume, making the estimation of the dose a matter to be settled once for all, instead of doing it for each tree covered.

\section{PROCEDURE.}

The cost of fumigation, and therefore the profit in its use, depends in a great measure upon the arrangement of details, especially in the economical use of time. This is more important than in the case of most methods for killing insects, because of the time, from forty to fifty minutes, required for the operation of the gas. Fumigation may be economically done in one of two ways: with a small outfit arranged to fit in with other work, or with a large number of tents sufficient to keep all hands busy.

Work with a small outfit can be arranged so as to waste but little time. Fortunately, the tent may, if desired, be left on all night without danger, so that a strict record of the time is not necessary, only that it be not too short. A good arrangement is as follows: The tents are placed on at the close of the day's work; they are changed after supper, and again just before bedtime, leaving them on until morning, care being taken to pull them off before the sun gets at them. This will give three fumigations each night.

Large outfits are so expensive that the owner generally feels like keeping them in operation all night, although some are used only in the evening. The number of tents necessary will depend on the size of the tent and the number of men. The smallest number of men that can work to advantage is two; they could handle perhaps twenty tents of medium size. This would allow two minutes for each tent, which ought to be sufficient to change the tent and introduce the chemicals. It is doubtful, however, if the fumigator should take part in the vigorous physical work of changing the tents where so much depends on his judgment. With the new method of determining the dose, described below, this does not hold, and two-man outfits should be entirely practical.

The number generally employed in a fumigating gang is four or five, according to the size of the trees. One man introduces the chemicals, another looks out for the generator and measures the acid, and two or three handle the tents. Such a gang can handle from thirty to forty medium-sized tents and cover from four to six acres of orchard in a night.

There is much variation in the detail of procedure in fumigating; one of the best methods is illustrated in the accompanying diagram, Fig. 19 . It is intended to represent a gang of four working with sheet tents in 
an orchard of rather small trees. Three trees are shown newly covered, one tent in the process of being shifted, and three others ready to have the tents removed. The four dark spots indicate the position of the men at the time the fumigator is about to go under the tent, and the lines of arrows show the paths over which the men will travel during the next minute. The fumigator enters the tent (Fig. 21), introduces the chemicals and quickly withdraws, sees that the tent is down tightly to the ground, and picks up his tray of cyanide and proceeds to the next tree. The helper holds up the edge of the tent while the fumigator enters, and drops it as he comes out, then turning he chirps to the horse in the acid
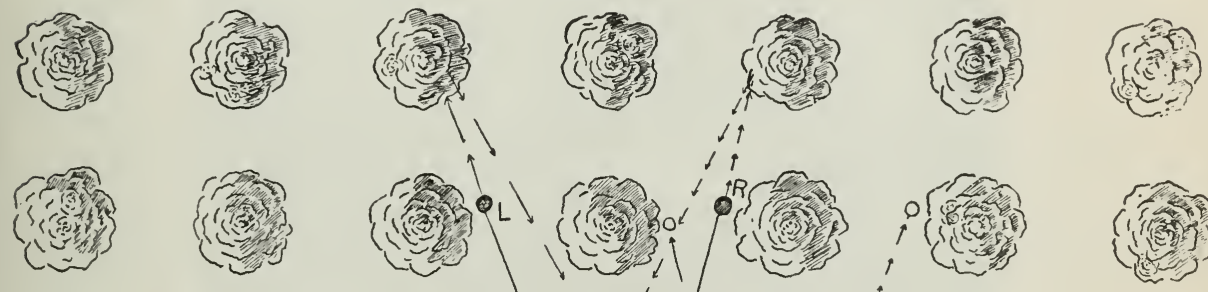

$\checkmark f$
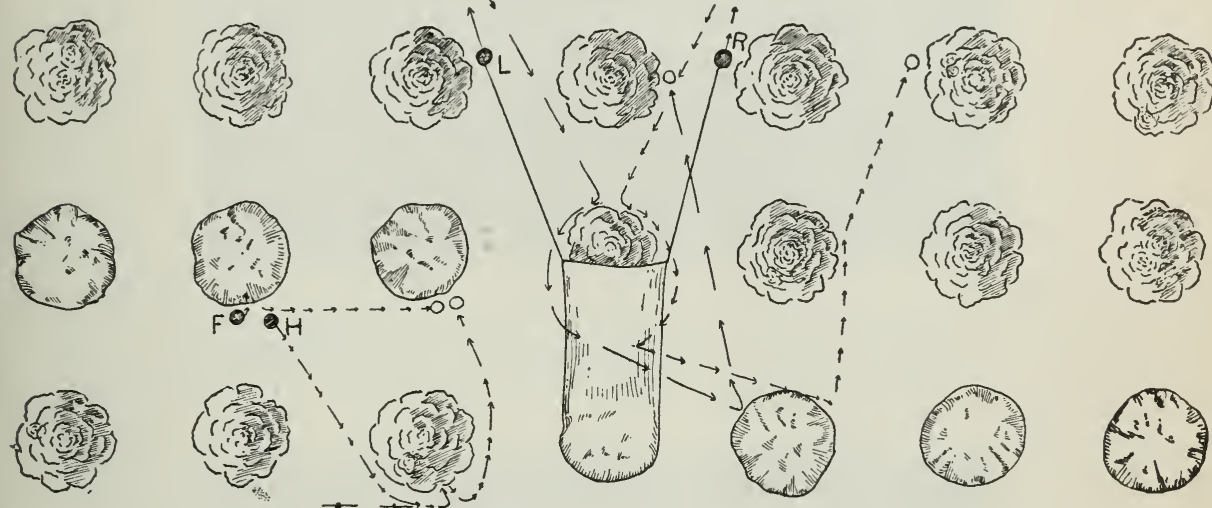

$\vdots$
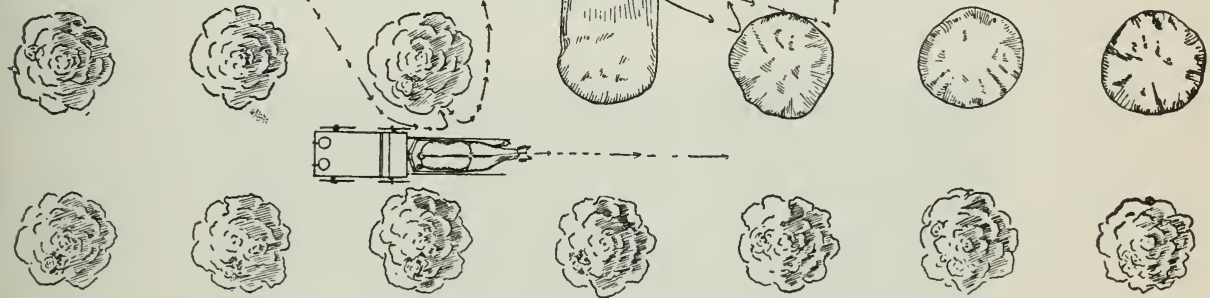

FIG. 19. Bird's-eye view of a young orchard being fumigated under sheet tents. F, the fumigator; $\mathbf{H}$, the helper who carries the generators and measures the acid; $\mathbf{R}$ and $\mathbf{L}$, the tent men. The arrow lines indicate the path traversed by each of these parties.

wagon and drives along to the next tree. He next removes the generator from beneath this tree, pours out the contents (already used) on the ground, measures out the water and then the acid according to the directions of the fumigator. (Fig. 20.) He then carries the generator to the fumigator, who is waiting by the next tent.

The tent men are shown in diagram (Fig. 19) as they are backing off with the end of the rope. They continue to back off as indicated until the tent is over the tree, then proceeding to the tent they adjust the bottom as they pass around it, one on either side. Proceeding to the next tent they attach their poles, then going to the other end of their poles, hold them down with their feet as they pull on their ropes and 
raise the edge of the tent. As soon as the pole is high enough they back off, and the ends of the lines show the position corresponding with that they had at first. The variations depend on differences in the plan of estimation, and in the kind of tent.

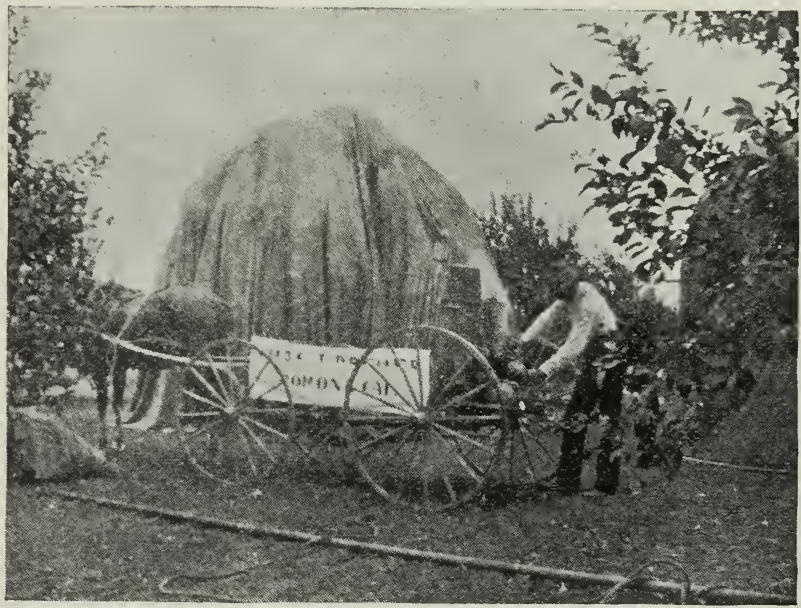

FIG. 20. THE A:ID WAGON-The helper in the act of measuring the acid in a graduate. A generator is shown on the shelf below the water tank. The torch is shown above and between the acid and water tanks.

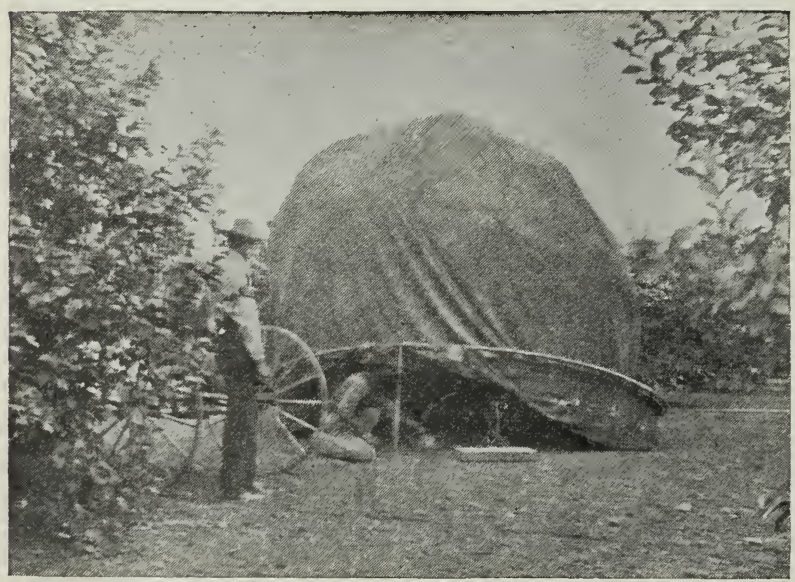

FIt. 21. The Fumgator, in the aet of charging a generator under the hoop tent.

Estimating the Dose.-The responsibility in the whole process rests on the fumigator, for he is the one who chooses the quantity of the dose; in practice the amount prescribed has depended wholly upon his personal judgment. The fumigator looks at a tree and says eight ounces, six and 
a half ounces, or ten ounces, according to his idea of its size. The result is that a great deal of unsatisfactory work is done. The wonder is, that the results are as uniform as they are. There are two ways of verifying one's judgment as to the proper doses to be given. The one most commonly used is a subsequent inspection of the trees; the practice being to give a little more gas than the trees will stand without injury. If the slight injury produced is the same on all the trees, the fumigator's judgment is supposed to be working normally. This is very misleading, for the larger the tree the greater the injury, if the dose is properly proportional to the cubic content. The reason for this is the difference in the generation of the gas in large quantities, and its relatively slower diffusion in a large volume.

The other method is to measure the tree and find the amount of the dose by consulting the tables. If the tables are correctly calculated and the measurement accurately done, this is a safe method; but there are grave difficulties in the way of accurately measuring a tree. If not tented, it is difficult to judge how much to allow for the bending of the branches under the weight of the tent. If tented, which is really the correct way, there are practical difficultios in measuring the diameter and height. To simplify the measurement and estimation of a tent the following table, based upon volume, was prepared by the author. The center column gives the various doses corresponding to the sizes of trees given in the columns on either side. Those on one side have been calculated so as to give three parts of hydrocyanic acid gas in 1000 parts of air (or 0.3 per cent), on the other side two parts in 1000 of air (or 0.2 per cent). For winter treatment for deciduous trees, 0.3 per cent gas is suggested, and is nearly the strength recommended in the Eastern States for the San José scale. One half of this amount is not far from the commonest practice in this State for citrus trees. The 0.2 per cent gas is suggested for citrus trees, and agrees with the amounts used by some of the most successful fumigators, although others get fair results with scarcely more than half this amount.

The measurements to be taken when using this table are, (1) around the tent, and (2) over the tent from ground to ground. If these two measurements are about equal, as they will be for many orange trees, the number nearest the measurement is found in the circumference column, and the corresponding dose will be seen in the center colunn. If these two measurements are not nearly the same, the outside columns become of use, for they show for each size how much difference must occur to make necessary a half ounce increase or decrease in the dose. That is, for each differential there must be added or deducted one half ounce of cyanide. For instance, if the difference between the distance over and around the tree is 5 feet, and the differential for that circumference is 3 feet 11 inches, then the dose must be increased or dinin- 
ished by a little more than one half ounce; but if that differential be 1 foot, then for each foot there must be added or subtracted one half ounce, or $2 \frac{1}{2}$ ounces for the 5 feet.

Table Showing Doses Suitable for Trees of Different Measurements.

\begin{tabular}{|c|c|c|c|c|c|c|c|c|}
\hline \multicolumn{4}{|c|}{ 0.3 Per Cent Gas. } & \multirow{2}{*}{ CYANIDE. } & \multicolumn{4}{|c|}{0.2 PER CENT GAS. } \\
\hline \multicolumn{2}{|c|}{$\begin{array}{c}\frac{1}{2} \text { Ounce } \\
\text { Differential. }\end{array}$} & \multicolumn{2}{|c|}{$\begin{array}{c}\text { Circumference of } \\
\text { Tree. }\end{array}$} & & \multicolumn{2}{|c|}{$\begin{array}{c}\text { Circumference of } \\
\text { Tree. }\end{array}$} & \multicolumn{2}{|c|}{$\begin{array}{l}\frac{1}{2} \text { Ounce } \\
\text { Differential. }\end{array}$} \\
\hline $\begin{array}{c}F t . \\
3\end{array}$ & $\begin{array}{l}\text { In } \\
5\end{array}$ & $\begin{array}{l}F t . \\
19\end{array}$ & $\begin{array}{l}I n . \\
1\end{array}$ & $\begin{array}{l}\text { Ounces. } \\
\quad 2\end{array}$ & $\begin{array}{l}F t \\
22\end{array}$ & $\begin{array}{l}\text { In. } \\
1\end{array}$ & $\begin{array}{c}F t . \\
3\end{array}$ & $\begin{array}{l}\text { In. } \\
11\end{array}$ \\
\hline 3 & & 20 & 6 & $2 \frac{1}{2}$ & 23 & 7 & 3 & 4 \\
\hline 2 & 7 & 21 & 11 & 3 & 25 & & 2 & 11 \\
\hline 2 & 4 & 23 & & $3 \frac{1}{2}$ & 26 & 4 & 2 & 8 \\
\hline 2 & 2 & 24 & 1 & 4 & 27 & 7 & 2 & 6 \\
\hline 1 & 11 & 25 & 1 & $4 \frac{1}{2}$ & 28 & 9 & 2 & 4 \\
\hline 1 & 10 & 26 & & 5 & 29 & 10 & 2 & 2 \\
\hline 1 & 9 & 26 & 10 & $5 \frac{1}{2}$ & 30 & 9 & 2 & \\
\hline 1 & 8 & 27 & 8 & 6 & 31 & 8 & 1 & 10 \\
\hline 1 & 7 & 28 & 5 & $6 \frac{1}{2}$ & 32 & 7 & 1 & 9 \\
\hline 1 & 6 & 29 & 1 & 7 & 33 & 4 & 1 & 8 \\
\hline 1 & 5 & 29 & 9 & $7 \frac{1}{2}$ & 34 & & 1 & 7 \\
\hline 1 & 4 & 30 & 4 & 8 & 34 & 8 & 1 & 6 \\
\hline 1 & 3 & 31 & 6 & 9 & 36 & 1 & 1 & 5 \\
\hline 1 & 2 & 32 & 7 & 10 & 37 & 5 & 1 & 4 \\
\hline 1 & 1 & 33 & 8 & 11 & 38 & 7 & 1 & 3 \\
\hline 1 & & 34 & 8 & 12 & 39 & 10 & 1 & 2 \\
\hline & 11 & 35 & 7 & 13 & 40 & 11 & 1 & 1 \\
\hline & 10 & 36 & 6 & 14 & 41 & 11 & 1 & 1 \\
\hline & 10 & 37 & 5 & 15 & 42 & 10 & 1 & \\
\hline & 9 & 38 & 3 & 16 & 43 & 9 & & 11 \\
\hline & 9 & 39 & & 17 & 44 & 8 & & 11 \\
\hline & 8 & 39 & 9 & 18 & 45 & 7 & & 10 \\
\hline & 8 & 40 & 5 & 19 & 46 & 5 & & 10 \\
\hline & 7 & 41 & 2 & 20 & 47 & 3 & & 10 \\
\hline & 7 & 42 & 8 & 22 & 48 & 9 & & 9 \\
\hline & 7 & 43 & 11 & 24 & 50 & 2 & & 9 \\
\hline & 6 & 45 & & 26 & 51 & 6 & & 8 \\
\hline & 6 & 46 & 1 & 28 & 52 & 8 & & 8 \\
\hline & 6 & 47 & 2 & 30 & 54 & & & 7 \\
\hline & 5 & 48 & 2 & 32 & 55 & 3 & & 7 \\
\hline
\end{tabular}

As an example, suppose a tree were 35 feet around and 36 feet over the top, and a person were using the 0.2 per cent table: Running down the circumference column we find that 34 feet 8 inches (the nearest to 35 feet) requires 8 ounces and that the differential is 1 foot 6 inches, that is, 35 feet requires a little over 8 ounces, and the 
difference between the two measurements around and over the tree, 1 foot, is nearly enough to require another half ounce, so that $8 \frac{1}{2}$ ounces would be about right. Suppose, again, the distance around a tree to be 40 feet, and that over the top only 35 feet; using the same table, we find opposite 39 feet 10 inches (the nearest to 40 feet) the dose 12 ounces. But the distance over the top is 5 feet less, and a less amount of cyanide will be necessary. We therefore use the differential (1 foot 2 inches) and deduct one half ounce for each 1 foot 2 inches difference, or about 2 ounces altogether. This leaves 10 ounces as the correct dose for this tree.

Method of Measurement.-In making these measurements the method which seems most feasible is that used in studying the fumigation

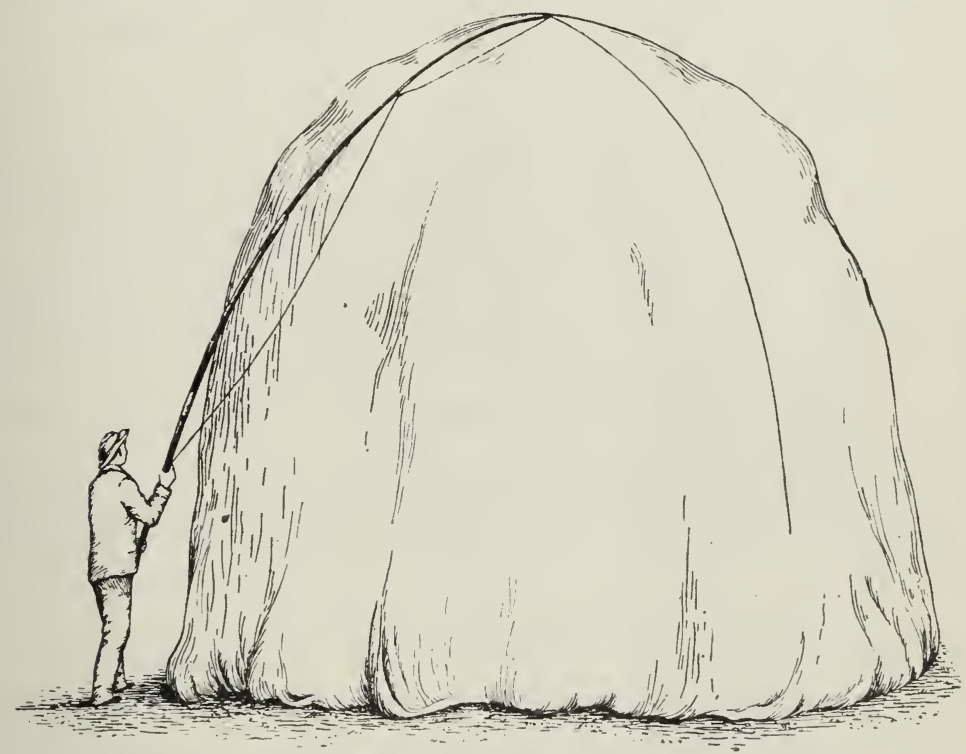

FIG. 22 .

dosage reported in Bulletin No. 152 of this Station. The apparatus used consisted of a fishing rod and wire line. A piece of electric drop light insulated cord was used as the line. This consists of a cable of many fine copper wires covered with thread, which gives greater flexibility and is less liable to kink than a simple wire. The line was marked off into one-meter (39.3 inches) lengths by knots; the metric system was used because of convenience in calculation. The rod employed was the ordinary jointed fishing-rod, which could be disconnected for transportation and adjusted to different heights of trees. The sections of the rod were marked off into centimeters for use in connection with the line in making the measurements.

The procedure in taking the measure of a tent is as follows: Having 
first attached the line at about its middle to the end of the rod, one end of the former is made fast to the tent. The most convenient way to accomplish this was found to be by means of a hook, like a fish-hook from which the barb had been removed. ' The most convenient place of attachment was at a point one meter from the ground. After attaching one end of the line to the tent the rest of that half is caused to lie up to and over the center and top of the tent by means of the rod. The one making the measurement then walks around to the opposite side of the tent, rod in hand, holding the line constantly in position over the top. The other end of the line is carried around the tent at the same time and is then drawn taut, measuring the last fraction of meter by means of the graduations on the lower joint of the rod. Adding now one meter, the distance the first end is from the ground, we have the measurement of the distance over the top of the tent from the ground on one side to the ground on the other.

A second measurement was then taken by throwing the line off the top of the tent by means of the rod and holding it so that as the measurer proceeds around the tent to the point where the line is attached it will encircle the tent at a point about one meter from the ground. The end of the rod is again brought into requisition and the last fraction of meter read in centimeters. Both measurements are thus made by one person in a single trip around the tent.

These measurements should be made with every tree, as it seems to be physically impossible to guess accurately.

The accompanying diagram (Fig. 23) will enable one to determine the volume of a tent from these measurements or from height and diameter.

New Dosage System.-In Bulletin No. 152 was suggested a dosage system corresponding more nearly to the actual practice than one based simply upon the volume of the tent. This is one proportional to the area of the canvas, and is suggested because with the tents now mostly in use the leakage of the gas is the most important item. The following table will show how much this scheme varies from previous recommendations:

\begin{tabular}{|c|c|c|c|c|c|c|c|}
\hline \multicolumn{8}{|l|}{ Size of tent, in meters- } \\
\hline Distance over tent & 13.73 & 10.86 & 8.64 & 6.87 & 5.40 & 4.32 & 3.39 \\
\hline Distance around the tent ..... & 18.30 & 14.48 & 11.52 & 9.16 & 7.20 & 5.76 & 4.52 \\
\hline Volume of tent, in steres...... & 108 & 56 & 28 & 14 & 7 & 3.5 & 1.75 \\
\hline \multicolumn{8}{|c|}{ Dose, in ounces- } \\
\hline By Morse schedule & 25.6 & 12.8 & 6.4 & 3.2 & 1.6 & .8 & .4 \\
\hline Present suggestion & 12.7 & 8.9 & 6.2 & 4.3 & 3.1 & 2.2 & 1.1 \\
\hline
\end{tabular}

New Scheme of Measuring.-In order to avoid the use of tables or of measuring apparatus we have suggested the marking of the dosage on the tents. This system consists in making a series of parallel lines near 
two opposite edges of the tent, which are so distanced from the center point that they shall correspond with the dosage of a tree of the average

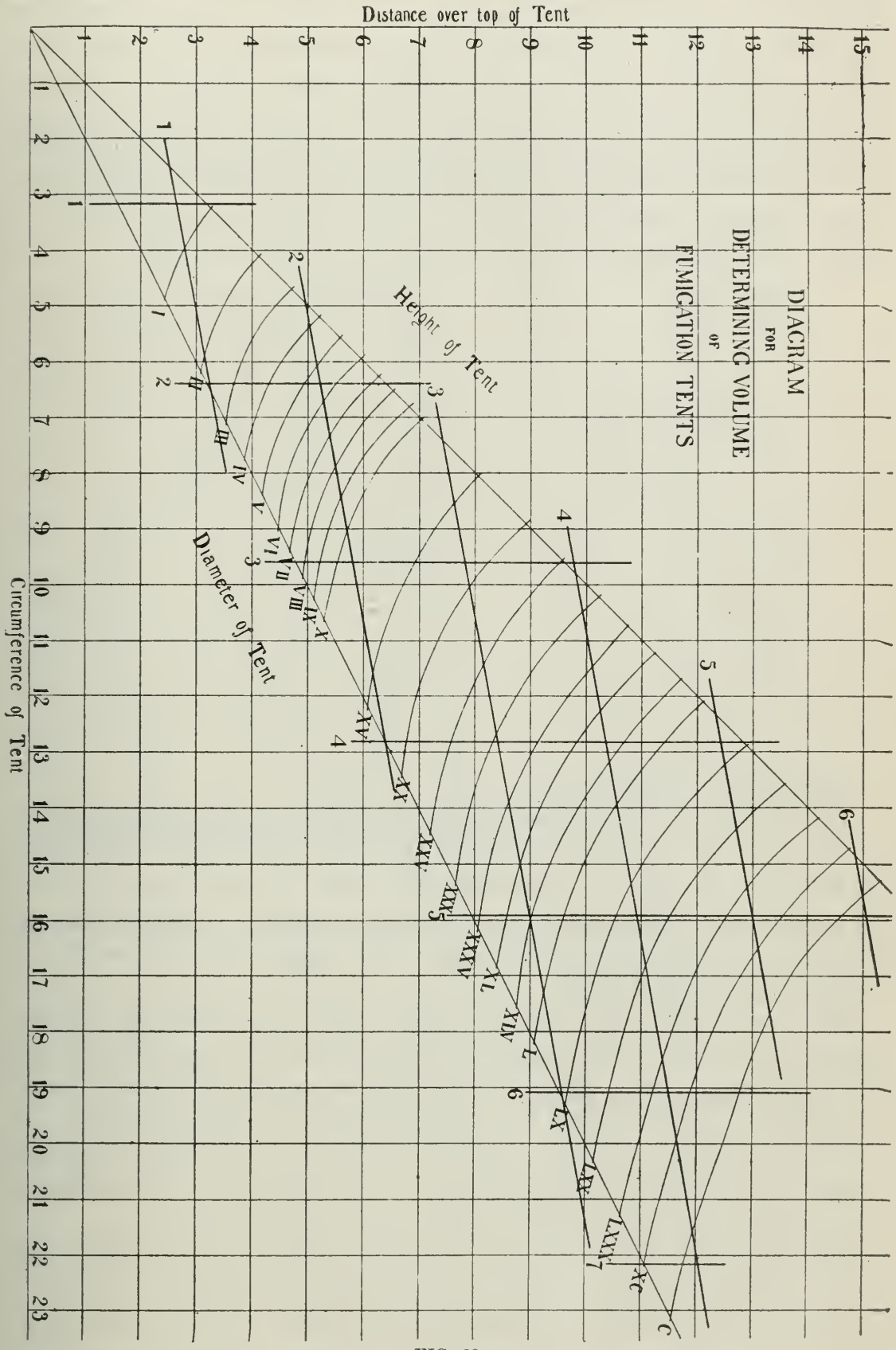

FIG. 23.

shape. Upon these lines will be placed numerals indicating the dose, the circumference in yards (paces), and the difference (that is, the 
amount the dose must be varied), should the distance around be more or less than the amount indicated for an average tent. This plan would permit the estimation of the dosage with sufficient rapidity and a fair degree of accuracy. If the tent is not put on the tree so that its center is directly over the center of the tree, the same dosage line may not touch the ground on the two sides, but the average of the two will give approximately the correct measurement. A lateral displacement will give no trouble of using the one indicating the highest dosage, if the rule is followed when more than one line touches the ground on one side. The following table gives the scheme of marking tents:

\begin{tabular}{rrrcc}
$\begin{array}{r}\text { Distance from } \\
\text { Center of Tent. } \\
\text { Ft. }\end{array}$ & In. & \multicolumn{3}{c}{ Numerals to be marked on the lines. } \\
2 & 5 & Ounces. & Paces. & Difference. \\
3 & 1 & $\frac{1}{2}$ & 3 & .1 \\
5 & 3 & 1 & 4 & .2 \\
6 & 5 & $1 \frac{1}{2}$ & 5 & .3 \\
7 & 6 & 2 & 6 & .3 \\
8 & 5 & $2 \frac{1}{2}$ & 7 & .4 \\
9 & 3 & 3 & 8 & .4 \\
10 & & $3 \frac{1}{2}$ & 9 & .4 \\
10 & 8 & 4 & 10 & .4 \\
11 & 4 & $4 \frac{1}{2}$ & 11 & .4 \\
12 & 8 & 5 & 12 & .4 \\
13 & 10 & 6 & 13 & .5 \\
14 & 11 & 7 & 14 & .5 \\
16 & & 8 & 15 & .5 \\
17 & & 9 & 16 & .6 \\
18 & & 10 & 17 & .6 \\
18 & 11 & 11 & 18 & .6 \\
& & 12 & 19 & .7
\end{tabular}

This whole series of lines would not be all placed on any one tent ordinarily, but only those useful for the given size of tent. Thus, for a 20 -foot tent the lines corresponding to a dose of $1 \frac{1}{2}$ to 4 ounces would be useful; for a 30 -foot tent, $2 \frac{1}{2}$ to 8 ounces, and for a 40 -foot tent, 4 to 12 ounces would be about the range. The appearance of these lines on a tent is shown in Fig. 24.

The use of the tent will be evident from the following example: Suppose the lines on the ground at the two sides of a tree showed 7 and 9 ounces, respectively, and the number of steps around the tent was 17 . The correct dosage would be 9 ounces. The average between 7 and 9 would have indicated 8 ounces, but the distance around was two steps more than 15, the number opposite the 8 ounces, and the difference that should be added for each of these is 0.5 of an ounce.

Estimating the size of the tree is usually done by one of three plans. Some persons plot the orchard in the daytime, indicating the dose for each tree, and fumigating at night in accordance with this prearranged plan. They claim, with some show of truth, that they are better acquainted with the trees by daylight and can more accurately estimate 
their size. Others do this at night, a row at a time; maintaining that, with practice, it can be done with as much accuracy as when done in the daytime, and that the danger of mistaking the rows is lessened. The third plan, which seems the most accurate, and the only one that can be recommended, is to make the estimate after the tent is on the tree. The weighing of the cyanide is done at night as a rule, but those working by the third plan generally have it weighed in the daytime.

When the weighing is to be done at night, a base of supplies is estab-

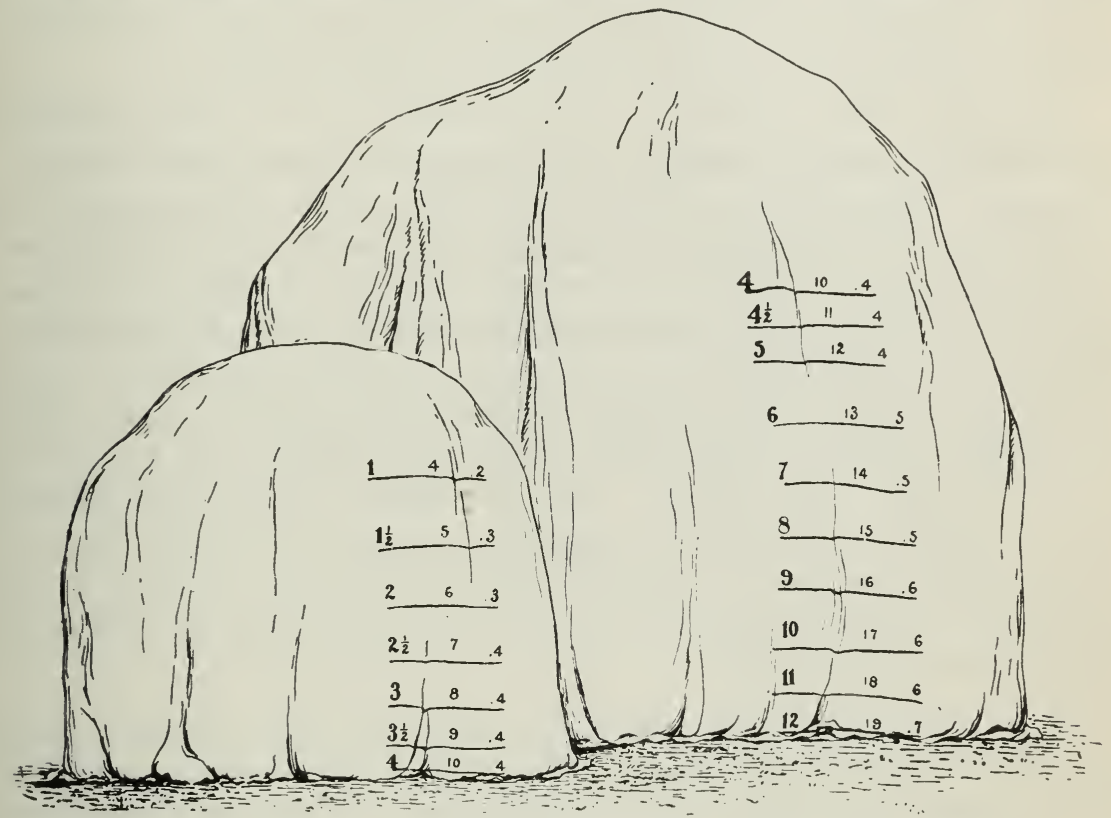

FIG. 24 .

lished as near the center of the field as possible, and the cyanide and acid, as well as the water, are dispensed at that point, the generators being carried there on trays. A generator tray is a frame holding four generators in a row, or eight if stacked two deep; a person can carry two trays, or generators enough for sixteen tents. After the chemicals are ready, the "fumigator" takes up his tray of cyanide, and the helper two trays of generators, and they proceed to tent after tent, leaving and charging a generator at each tent. When half-way along the row, the helper drops a tray that has been emptied; when he reaches the other end, both trays are empty. The generators are arranged on the trays, as are also the doses of cyanide on the fumigator's tray, in the order of the trees. The next work for the fumigator is to estimate another row of trees, and while he is doing this, the helper gathers up the generators of the previous row. They soon both arrive at the base of supplies and proceed to measure and weigh the chemicals for a row 
on the other side. The details of the third method have already been lescribed.

When the weighing is done in the daytime, the average dose is commonly weighed into each can or bag, and a little added or subtracted from the dose as the size of the tree may indicate. When the trees are very uniform, the dose thus varying but little, this may do very well; but if the variation is greater, it will be well to have different sized (loses weighed out. When this is done the can or bag should indicate clearly the amount of its contents by its different shape or character. 'This method seems distinctly preferable to night weighing.

Charging.-The generator now universally used is the ordinary earthenware vessel or chamber, the cheap yellow ware being generally selected. Some use a perforated sheet-lead cover, but generally no cover is used. The fumigator places the generator on the ground near the trunk of the tree and is then ready to charge it, which he accomplishes by dropping the cyanide into the diluted acid in the generator. He has the cyanide either in tin cans or in small paper bags; in the former case he pours the cyanide into the acid, keeping the can, which he replaces in his tray, but in the latter case breaks the bag and drops it into the acid, bag and all. Some fumigators prefer to add the acid last, in which case the helper brings the generator with only water in it, and keeps the acid in a small pitcher, one for each generator. In this case the fumigator puts the cyanide in the generator, as before, and then pours in the acid.

Poisonous Nature of the Gas.-All the work of the fumigator under the tent is done at arm's length. There is no poison more dangerous or fatal than hydrocyanic acid. The danger from the gas is greatest as it is coming up from the generator. This is so well understood that though the gas has been used for years by a great many people, we have never heard of an accident with it. There seems to be no injurious effect from breathing the diluted gas that fills the air when the tents are removed, even though it may smell very strong and one can feel it very plainly in his throat and chest. Working every night, for months at a time, does not develop any abnormal symptoms, so it can be safely said that, with proper care, there is no particular danger in the use of the gas.

Inspection.-Wherever fumigation is carefully done the tents will be thoroughly inspected every day. To do this the inspector goes beneath the tent as it lies on the ground, and any holes will be at once seen by the light streaming through; these places are marked and patches applied. Sometimes the patch is glued on, but the usual and preferable way is to sew it on. Sewing is done by hand in the same way as sails are mended, or sometimes a sewing-machine is used. 


\section{CONCLUDING REMARKS.}

The uniform testimony of those who have used fumigation extensively is that by no other means at any cost can as effective work be done as by proper fumigation. It is also true that at no place where fumigation has been followed for years is it believed that the method is eradicative. Some, indeed, think that it would be if universally used, but those longest acquainted with the process do not claim that for it. When it is well clone, the insects that escape are far below one per cent; but with insects capable of increasing several hundred per cent in a single season, the destruction of over ninety-nine per cent is certainly not a bad result.

The question between fumigation and spraying will usually resolve itself into this: If the interest of the tree or crop demands a degree of freedom from scale insects that can not be insured by one or two sprayings, fumigation will be resorted to. The cost of cyanide has been reduced about one half since fumigation was begun, and if it should be reduced to about one half of what it is now, fumigation would probably entirely take the place of spraying for scale insects on all trees, as it has now done to such an extent in the case of citrus trees that are so difficult to spray. 


\section{REPORTS AND BULLETINS AVAILABLE FOR DISTRIBUTION.}

\section{REPORTS.}

1896. Report of the Viticultural Work during the seasons 1887-93, with data regarding the Vintages of $1894-95$.

1897. Resistant Vines, their Selection, Adaptation, and Grafting. Appendix to Viticultural Report for 1896.

1898. Partial Report of Work of Agricultural Experiment Station for the years 1895-96 and $1896-97$.

1900. Report of the Agricultural Experiment Station for the year 1897-98.

1902. Report of the Agricultural Experiment Station for 1898-1901.

\section{BULLETINS.}

No. 121. The Conservation of Soil Moisture and Economy in the Use of Irrigation Water.

125. Australian Saltbush.

127. Bench-Grafting Resistant Vines.

128. Nature, Value, and Utilization of Alkali Lands.

129. Report of the Condition of Olive Culture in California.

131. The Phylloxera of the Vine.

132. Feeding of Farm Animals.

133. Tolerance of Alkali by Various Cultures.

134. Report of Condition of Vineyards in Portions of Santa Clara Valley.

135. The Potato-Worm in California.

136. Erinose of the Vine.

137. Pickling Ripe and Green Olives.

138. Citrus Fruit Culture.

139. Orange and Lemon Rot.

140. Lands of the Colorado Delta in Salton Basin, and Supplement.

141. Deciduous Fruits at Paso Robles.

142. Grasshoppers in California.

143. California Peach-Tree Borer.

144. The Peach-IVorm.

145. The Red Spider of Citrus Trees.

146. New Methods of Grafting and Budding Vines.

147. Culture Work of the Substations.

148. Resistant Vines and their Hybrids.

149. California Sugar Industry.

150. The Value of Oak Leaves for Forage.

151. Arsenical Insecticides.

152. Fumigation Dosage.

153. Spraying with Distillates.

154. Sulfur Sprays for Red Spider.

155. Directions for Spraying for the Codling-Moth.

Copies may be had by application to the Director of the Experiment Station, Berkeley, California. 